\title{
"No te conozco, pero aquí estoy" Cartas solidarias en tiempos de COVID-19 en España
}

\section{"No te conozco, pero aquí estoy" Solidarity letters in times of COVID-19 in Spain}

\author{
Pilar González-Cerveraa ${ }^{a}$ Rubén Mirón-González ${ }^{b}$ \\ a Hospital Universitario La Paz, Madrid, España \\ b Departamento de Enfermería y Fisioterapia, Grupo de investigación Cuidado Comunitario y Determinantes Sociales de la Salud, \\ Universidad de Alcalá, España
}

\section{Resumen}

Introducción: Abril de 2020 concluyó con más de 80.000 pacientes hospitalizados por COVID-19 en España. El aislamiento social es uno de los problemas derivados de la institucionalización. Para combatir este aislamiento social surgió el proyecto "No te conozco, pero aquí estoy" (NTCPAE), que tuvo como objetivo la recepción y el envío de al menos 6.000 cartas en 36 hospitales y 6 residencias de España. Objetivos: Identificar cuál fue la motivación de los remitentes de las cartas a participar en el proyecto NTCPAE. Metodología: Estudio transversal, observacional y descriptivo. Se diseñó un cuestionario de 19 preguntas de elaboración propia y se mandó a 4.820 remitentes. Resultados: Se han recibido 2.021 cuestionarios, de los cuales el 89,4\% fueron mujeres. El 55,4\% refirieron tener una persona cercana afectada por COVID-19 y las preguntas obtuvieron una puntuación mayor de 3,8 puntos sobre 5. Discusión: Los encuestados mostraron una motivación a participar en el proyecto relacionados con la empatía, la satisfacción personal y la repercusión positiva en la salud emocional de la persona institucionalizada con COVID-19. Conclusiones: Consideramos que el proyecto NTCPAE ha ayudado a materializar la motivación y el compromiso social de la población en beneficio de la persona institucionalizada durante la pandemia de COVID-19.

Palabras clave: solidaridad; conducta de ayuda; individualismo; incertidumbre; vulnerabilidad social; carta; escritura; COVID-19.

\section{Abstract}

Introduction: April 2020 concluded with more than 80,000 COVID-19 patients hospitalized in Spain. Social isolation is one of the problems derived from the institutionalization. To combat this problem, the project "No te conozco, pero aquí estoy" (NTCPAE) -I don't know you but here I am- emerged, which aimed to receive and send at least 6,000 letters in 36 hospitals and 6 nursing home. Objectives: To identify the motivation of the senders of the letters to participate in the NTCPAE project. Methodology: Cross-sectional, observational and descriptive study. We have designed a 19 self-prepared items questionnaire and we sent 4,820 questionnaires. Results: We have received 2,021 fulfilled questionnaires, of which $89,4 \%$ were women. $55,4 \%$ reported to have had a close person with COVID-19, and the questions scored higher than 3,8 points out of 5 . Discussion: Respondents showed a motivation to participate in the project related to empathy, personal satisfaction and the positive impact on emotional health of the institutionalized person with COVID-19. Conclusions: We consider that the NTCPAE project has helped to materialize the motivation and social commitment of the population for the benefit of the institutionalized person during the COVID-19 pandemic.

Keywords: solidarity; helping behaviour; individuation; uncertainty; social vulnerability; letter; writing; COVID-19. 


\section{Introducción}

E n las últimas décadas, nuestra forma de relación y cultura han estado marcados por un constante cambio. En este sentido, el modelo social de Modernidad Líquida de Zygmun Bauman (1925-2017) ha supuesto un impacto en el análisis de nuestras sociedades. Este autor pone de manifiesto que los vínculos interpersonales se han dejado atrás y que el individualismo, el consumo del bienestar y el hedonismo, entre otros, justifican hoy en día la manera de relacionarnos (Bauman, 2005).

Durante la pandemia del COVID-19, hemos visto como la moralidad individual ha puesto en el punto de mira las dimensiones política, económica, social y cultural. La sociedad esperaba que los Estados respondieran con una mayor coordinación, eficiencia y transparencia en el manejo internacional de la pandemia del COVID-19 (Arora et al., 2020). Ante esta carencia, ha surgido la acción comunitaria como respuesta para intentar combatir el miedo a la incertidumbre y asumir la perspectivas de otros (Qian et al., 2020).

Según el Ministerio de Sanidad, Consumo y Bienestar Social, España concluyó el mes de abril de 2020 con 203.715 casos positivos, 15.873 personas fallecidas y 81.627 hospitalizadas (Instituto de Salud Carlos III, 2020). Los efetos psicológicos del aislamiento ante la hospitalización durante la pandemia del COVID-19 todavía están por descubrir. Pero si tenemos en cuenta otros tipos de aislamiento en la literatura, podríamos encontrar algunos efectos como el aburrimiento, el aislamiento social, el estrés, la ansiedad o la depresión, entre otros (Mengin et al., 2020).

Bajo la motivación de la acción comunitaria y el objetivo de combatir el aislamiento social del paciente institucionalizado por COVID-19, surgió la iniciativa "No te conozco, pero aquí estoy" (NTCPAE). Se trata de un proyecto que se llevó a cabo entre marzo y abril de 2020, y contó con la participación de más de 40 jóvenes, entre 22 y 30 años, de distintos sectores profesionales de la Fundación Tatiana Pérez de Guzmán el Bueno de Madrid. NTCPAE consistió en la recepción de cartas anónimas de ciudadanos, en su mayoría españoles, y el envío a personas desconocidas ingresadas en hospitales o residencias por el COVID-19. La finalidad de esta iniciativa fue romper con el aislamiento social e intentar mejorar la salud emocional de los pacientes hospitalizados.

El proyecto epistolar, totalmente voluntario y altruista, estuvo dividido en cinco departamentos. El departamento de logística y tratamiento de datos creó una base de datos y coordinó el resto de los departamentos. El departamento de redes sociales generó una comunidad de 8.337 voluntarios y compartió la evolución del proyecto. El departamento de medios de comunicación trabajó en la captación de colaboradores a través de la televisión y la radio, nacional e internacional, así como en la conexión con hospitales de toda España. El departamento de buzón filtró el contenido de las cartas según criterios de calidad. Por último, el departamento de envíos expidió un total de 6.000 cartas a un total de 36 hospitales y 6 residencias de mayores de España.

Nos encontramos ante una iniciativa social sin precedentes en la literatura científica que tuvo su impacto en prensa nacional e internacional (Poulet, 2020). Rápidamente, en España se llevaron iniciativas similares bajo títulos como "No te conozco, pero puedo ayudarte" (Antena 3 Noticias, 2020), "No te conozco, pero estas palabras son para ti" (Castillo del, 2020), "Sigo aquí" (Figueredo, 2020) o "No te conozco, pero estamos juntos en esto" (Martín, 2020).

En el informe del Diagnóstico de la situación del voluntariado en España, se afirma que las intervenciones sociales "son un conjunto de acciones intencionadas para mejorar el contexto de un colectivo en un territorio determinado que propician un progreso o cambio social valorado positivamente, tanto por los que ofrecen su acción altruista como por los que reciben los beneficios" (Ministerio de Sanidad, Política Social e Igualdad, 2011, p. 37). En este aspecto, gracias a iniciativas sociales como NTCPAE, se ha permito conectar la sociedad y empatizar con grupos vulnerables a través de acciones altruistas, tratando de producir un impacto positivo en la sociedad.

\section{Objetivos}

Unavezconcluido el proyecto NTCPAE, nos preguntamos ¿qué es aquello que ha motivado a la población a enviar cartas a los pacientes institucionalizados por COVID-19? En esta línea, el presente trabajo tiene como objetivo principal identificar cuál ha sido la motivación de los remitentes de las cartas a participar en el proyecto 
NTCPAE. Como objetivos secundarios, hemos querido conocer las dificultades que han tenido y la percepción personal sobre la situación del COVID-19.

\section{Metodología}

Se ha realizado un estudio transversal, observacional y descriptivo. En primer lugar, los autores diseñamos un cuestionario de 19 ítems (véase Anexo). Las preguntas fueron de elaboración propia, ya que no encontramos ningún estudio o herramienta que nos pudiera guiar, y se elaboraron según la realidad que queríamos explorar. Se utilizaron preguntas dicotómicas, de escala Likert y de respuesta abierta. Fueron organizadas en los siguientes bloques temáticos: variables sociodemográficas (ítems del 1 al 5, véase Anexo), variables motivacionales (del 6 al 13), problemas o dificultades (14 y 15) y percepción sobre la situación del COVID-19 (del 16 al 19).

Una vez diseñado el cuestionario, se editó en la plataforma Wimadd y se envió por email a 4.820 participantes del proyecto NTCPAE, también se dio difusión por la cuenta Instagram del proyecto. Los datos fueron recogidos entre el 30 de abril y el 11 de mayo de 2020, una vez concluido el proyecto NTCPAE. Se trata de un estudio exploratorio que no pretende obtener resultados estadísticamente representativos.

Se garantizó el anonimato de los encuestados y se obtuvo su consentimiento informado para la utilización de los datos a través del cuestionario. El análisis cuantitativo se realizó con el programa Excel $\circledast$, consultando la media aritmética y la moda. El análisis cualitativo se realizó a través de las fases de Taylor y Bogan (1996): descubrimiento, codificación y relativización. Además, utilizamos el programa Atlas.ti 8 para conocer la moda de palabras utilizadas en las tres preguntas abiertas.

\section{Resultados}

\section{Perfil sociodemográfico}

La respuesta de participación ha sido del 41,9\% ( $n=$ $2.021)$, de la cual el $89,4 \%(n=1.801)$ han sido mujeres. El rango de participación por edades y sexo se puede observar en la Figura 1.

El $84,6 \%(n=1.710)$ de los participantes vivían en España. Las provincias de mayor participación fueron: Madrid (35,5\%, $n=718)$, Andalucía (10,8\%, $n$ $=214)$, y Cataluña $(9.8 \%, n=200)$, se observa una amplia dispersión geográfica. Respecto al nivel de estudios de los participantes: el $41 \%(n=830)$ tenían estudios universitarios, el $24,3 \%(n=493)$ bachillerato o formación profesional, el 18\% ( $n=365)$ máster universitario, el 12,3\% ( $n=250)$ educación secundaria obligatoria, el 2,6\% $(n=54)$ educación primaria y el $1,3 \%(n=28)$ doctorado.

El $55,4 \%(n=1.120)$ de los encuestados afirmaron tener alguna persona de su círculo cercano (familia, amigos y/o compañeros de trabajo) contagiado con COVID-19. Respecto a los resultados de las preguntas cerradas de escala Likert, vienen resumidos en la Tabla 1. Todos los ítems han sido evaluados por parte de los participantes con una puntuación mayor de 3,8 puntos sobre 5 (Tabla 1).

Respecto a la pregunta abierta 13, "¿Qué motivos te han llevado a identificarte con alguien que no conoces?", hemos establecido como tema principal el "amor al prójimo", teniendo en cuenta las categorías identificadas de solidaridad y empatía. En esta línea, hemos identificado respuestas como: "ponerme en su lugar y saber que está pasando por momentos difíciles", "ayudar a alguien, acompañarlo en su enfermedad para que no se sienta tan solo" o "compasión y ganas de acompañar teniendo en cuenta las limitaciones. Salir de mí misma para aportar mi granito de arena".

Respecto a la pregunta abierta 15, "¿Cuáles han sido tus dificultades para enfrentarte a este coronavirus?", han provocado la creación dos categorías: personas que alegan no haber tenido ningún tipo de dificultad y aquellas cuales el tema principal ha sido el sufrimiento manifestado a través de sentimientos como: el miedo, la incertidumbre, la ansiedad, aislamiento, el distanciamiento familiar y relacional, o la preocupación económica y/o laboral.

Respecto a la pregunta abierta 19, “¿Qué conclusiones sacas de esta enfermedad?" Se identifica una aceptación moral que no se tenía presente: la vulnerabilidad. Por otro lado, la importancia del valor de la vida, que se expresa en el refuerzo de vínculos sociales y amistosos, la importancia de la solidaridad y la comunidad. Por último, las personas hacen referencia a una mejora en el sistema sanitario y los campos de investigación (Figura 2). 


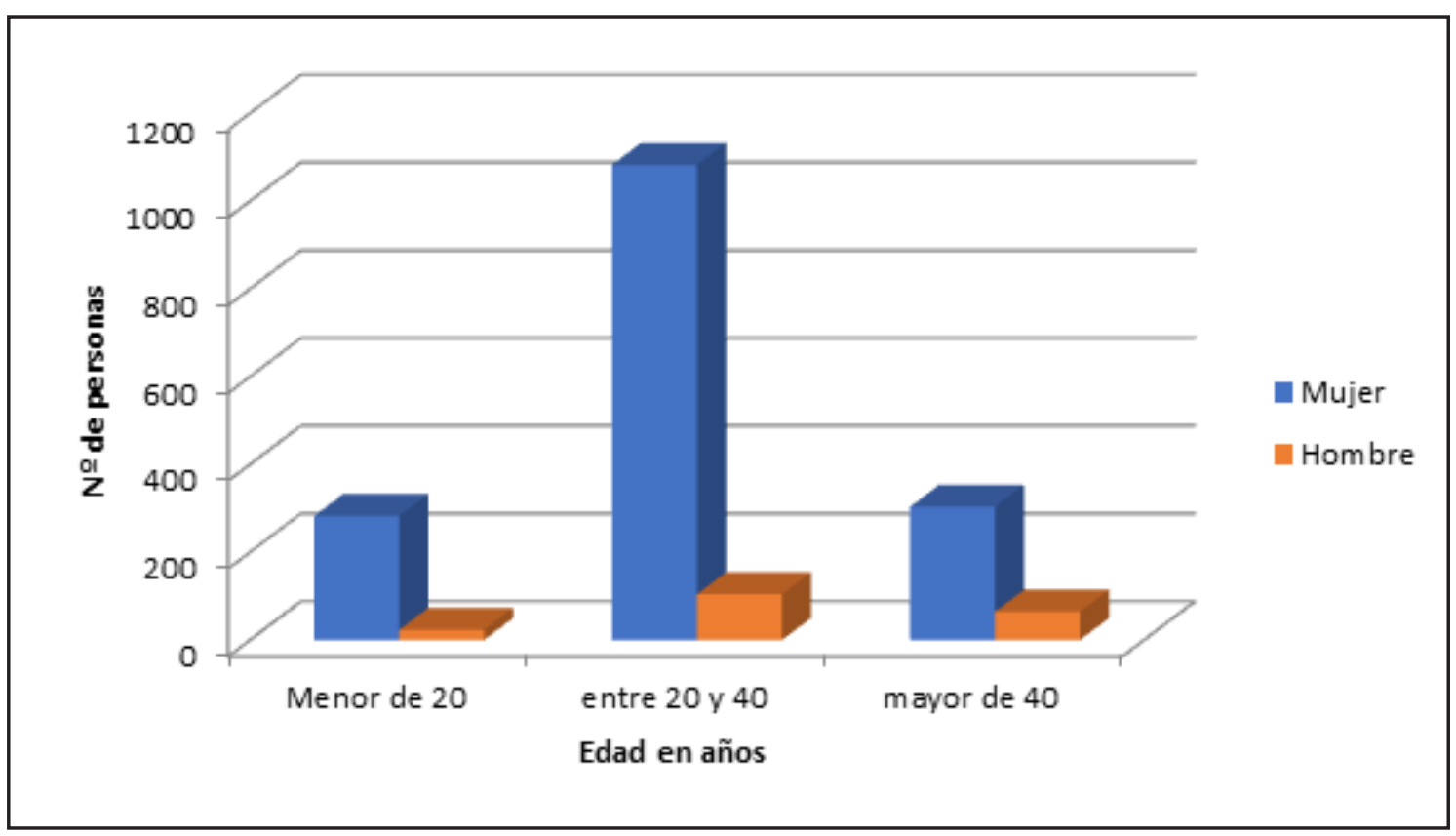

Figura 1. Rango de participación por edades y sexo.

Tabla 1. Resultados de las preguntas de respuesta cerrada con escala Likert (del 1 al 5).

\begin{tabular}{|c|c|c|}
\hline Variable & Ítem & Media \\
\hline \multirow[t]{6}{*}{ Motivación } & $\begin{array}{l}\text { ¿Crees que la sociedad civil tiene un papel importante para poder en marcha y } \\
\text { liderar proyectos solidarios? }\end{array}$ & 4,6 \\
\hline & $\begin{array}{l}\text { Tengo confianza en que las cartas que he enviado van a suponer un impacto } \\
\text { positivo en la salud mental del destinatario. }\end{array}$ & 3,8 \\
\hline & $\begin{array}{l}\text { Pensar que acciones pequeñas puede influir positivamente en la vida de } \\
\text { personas que no conozco, me motiva a buscar la manera de colaborar dentro } \\
\text { de mis posibilidades. }\end{array}$ & 4,7 \\
\hline & $\begin{array}{l}\text { Los beneficios de enviar cartas a pacientes con COVID-19 han supuesto una } \\
\text { motivación para ser una persona más comprometida socialmente. }\end{array}$ & 4,3 \\
\hline & $\begin{array}{l}\text { Estoy motivado en comprometerme con el envío de cartas a pacientes que } \\
\text { sufren aislamiento social. }\end{array}$ & 4,4 \\
\hline & $\begin{array}{l}\text { El envío de cartas a personas enfermas me ha supuesto una satisfacción } \\
\text { personal. }\end{array}$ & 4,6 \\
\hline $\begin{array}{l}\text { Problemas } 0 \\
\text { dificultades }\end{array}$ & $\begin{array}{l}\text { Garantizar escribir un mensaje adecuado dentro de mi carta ha supuesto } \\
\text { una dificultad sobre si escribía o no un contenido adecuado, partiendo del } \\
\text { desconocimiento de mi receptor. }\end{array}$ & 3,8 \\
\hline \multirow{3}{*}{$\begin{array}{l}\text { Percepción sobre } \\
\text { la situación del } \\
\text { covid-19 }\end{array}$} & $\begin{array}{l}\text { ¿Crees que esta pandemia nos ha permitido descubrir que somos socialmente } \\
\text { más vulnerables? }\end{array}$ & 4,5 \\
\hline & $\begin{array}{l}\text { Consideras que has obtenido un aprendizaje personal, social o moral sobre esta } \\
\text { pandemia mundial. }\end{array}$ & 4 \\
\hline & $\begin{array}{l}\text { Consideras que valoras algo más en tu vida personal que antes del confinamiento } \\
\text { pasadas por alto. }\end{array}$ & 4 \\
\hline
\end{tabular}




\section{cambiar vulnerables \\ debemos

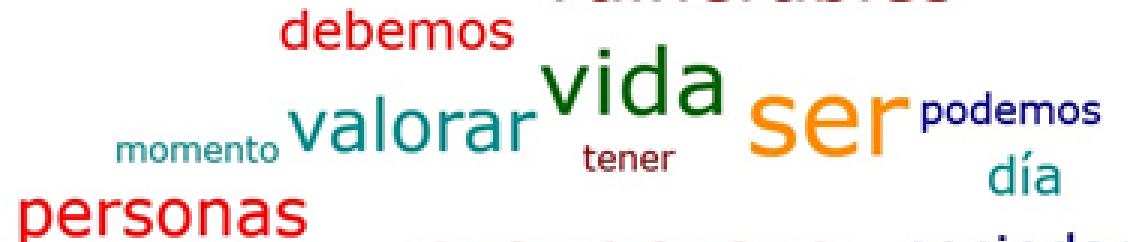 \\ gente temmo SOMOS sociedad \\ tenemos ${ }^{\text {puede }}$ dios vivir $_{\text {vin }}$

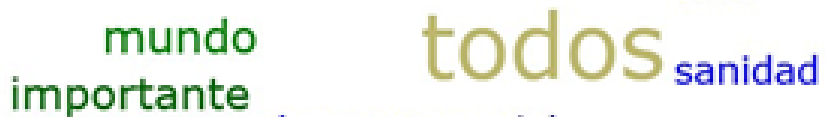 \\ humano social}

Figura 2. Palabras más utilizadas por los encuestados ante la pregunta "¿Qué conclusiones sacas de esta enfermedad?". (Figura generada con Atlas.ti 8).

\section{Discusión}

\section{Motivación para participar en el proyecto NTCPAE}

Como hemos visto, la mayoría de las remitentes de cartas fueron mujeres entre 20 y 40 años. Esta participación femenina coincide con la mayor presencia de mujeres en actividades solidarias o en relación con el compromiso social que propone la plataforma del voluntariado en España (Ministerio de Sanidad, Servicios Sociales e Igualdad, 2019, p. 14).

La mayoría de los participantes fueron de las Comunidades Autónomas de Madrid, Andalucía y Cataluña. Entre ellas identificamos las dos regiones con mayor número de casos positivos de COVID-19 en España a finales de abril de 2020: Madrid con 58.486 casos y Cataluña con 41.573 (Instituto de Salud Carlos III, 2020, p. 4). Además, más de la mitad de la muestra confirmaron haber tenido algún familiar, amigo o compañero laboral afectado por el COVID-19. En esta línea, podríamos entender que la población encuestada guardaba especial sensibilidad debido a la realidad que estaba viviendo.

La situación de aislamiento social del paciente con COVID-19 fue una motivación para participar en NTCPAE $(4,4$ puntos sobre 5$)$. Es posible que muchos de los encuestados estuvieran motivados ante la situación cercana de algún ser querido cercano, así queda manifestado en uno de los testimonios de los encuestados cuando se le preguntaba sobre la motivación que le había llevado a participar en el proyecto: "ayudar a alguien, acompañarlo en su enfermedad para que no se sienta tan solo".

Otra de las motivaciones identificadas para participar en el proyecto fue la propia satisfacción personal $(4,6$ puntos sobre 5), motivación intrínseca. Lipovetsky afirma que la solidaridad es una respuesta a una emoción del ser humano con respecto al sufrimiento y el dolor de los demás, sin que ello implique un interés superior (Lipovetsky, 1994 citado en: Giraldo Nayrobis \& RuizSilva, 2015), por lo que el sufrimiento ajeno y colectivo abre un espacio de implicación y colaboración al individuo.

El compromiso solidario, el considerar que la sociedad civil tiene un papel importante para poner en marcha proyectos solidarios y sentirse una persona más comprometida socialmente, obtuvo puntuaciones elevadas (4,6 y 4,3 puntos sobre 5 respectivamente). Los resultados podrían guardar relación con la visión de descoordinación que ha tenido la sociedad respecto la gestión del COVID-19 (Arora et al., 2020). Una carencia internacional que viene siendo denunciada desde epidemias como la del virus Zika o el Ébola (Pang, 2016), y donde Europa ha demostrado importantes deficiencias en lo que respecta a gestión de crisis sanitarias (Requeijo-Rey, Losada-Diaz, \& GarcíaJiménez, 2015). En este aspecto, se podría comprender que la sociedad quisiera colaborar, sentirse parte de la solución y cubrir posibles vacíos gubernamentales.

Respecto a los ítems que valoran una motivación extrínseca, es decir, generar un impacto positivo en la salud mental del destinatario e influir positivamente en la vida de personas desconocidas, han obtenido puntuaciones elevadas (3,8 y 4,7 puntos sobre 5 respectivamente). Esto podría guardar relación con el test de solidaridad realizado por Elsa Punset en España en 2012. En éste, concluyó que las situaciones que más felices hacen a las personas están relacionadas con los actos solidarios hacia personas desconocidas. Según palabras de Punset, "en tiempos de crisis, donde vemos que los gobiernos no llegan a todo, es fundamental despertar la capacidad activa de ayuda 
de las personas que forman parte de una sociedad. Nos necesitamos, y cada día más porque somos más, y para sobrevivir y prosperar tendremos que colaborar más" (Médicos del Mundo, 2012).

\section{Dificultades y vulnerabilidad}

Los encuestados han considerado que han tenido algún tipo de dificultad para escribir una carta adecuada al objetivo del proyecto (3,8 sobre 5 puntos). El confinamiento podría dificultar la visibilidad del problema. En este sentido, deberíamos reflexionar sobre la posible influencia que ha podido tener el Gobierno y los medios de comunicación sobre los ciudadanos. Según González Álvarez (2010) la comunicación social desempeña un rol importante en la sensibilización de la opinión pública y en los cambios de actitud de las personas de cara a la implicación y desarrollo con la sociedad. Además, como sanitarios consideramos todo un reto dirigir mensajes neutros o válidos para distintos perfiles de enfermos.

Respecto a las dificultades manifestadas por los encuestados, respecto al COVID-19 (pregunta 15), muchos han manifestado sufrimiento a través del miedo, la incertidumbre, la ansiedad, el aislamiento, el distanciamiento social, o la preocupación económica y/o laboral. Este sentimiento de sufrimiento podría tener relación con el sentimiento de vulnerabilidad, ya que implica fragilidad ante una situación de amenaza 0 un daño físico. En esta línea, la población encuestada ha reflejado una mayor sensación de vulnerabilidad como consecuencia del COVID-19 (4,5 sobre 5 puntos). Chambers, hace alusión a dos facetas que componen el sentimiento de vulnerabilidad: la exposición a tensiones y la dificultad para enfrentarse a ellas (Chambers, 1983, citado en: Feito, 2007). La exposición a tensiones podría estar relacionado con el dolor, el aislamiento, la soledad. Por otro lado, la dificultad para enfrentarse a la vulnerabilidad podría estar relacionado con la falta de información, el cumplimiento obligado de medidas de prevención, la parálisis de las rutinas o el freno a modelos de vida acelerados. Éstas son líneas que sería interesante explorar en el futuro.

\section{Percepción sobre la situación del COVID-19}

La población encuestada refiere haber adquirido un aprendizaje personal (4 sobre 5 puntos) y que actualmente valoraran más su vida personal que antes del confinamiento (4 sobre 5 puntos). Como se mencionaba anteriormente, la teoría de Bauman descubre que nuestra cultura dominante está inducida a un individualismo autorreferencial donde la libertad se convierte en un bien absoluto (Bauman, 2019). Las medidas de confinamiento obligatorio y la respuesta a la crisis, al implicar un compromiso colectivo que restringe y orienta el ejercicio de libertad, cuestionan las prioridades establecidas por las preferencias del individuo. Reajustar la conducta desde la anterior normalidad a un nuevo espacio de excepcionalidad $y$, posteriormente, su adaptación a una normalidad diferente, la llamada nueva normalidad, es un desafío especialmente complejo en sociedades fragmentadas y liquidas (Ministerio de Defensa, 2018).

\section{Conclusiones}

El envío de cartas ha impulsado una conducta humana hacia las personas más vulnerables. En este aspecto, Chivenato (2007, p. 48) nos explica que la conducta humana viene determinada por tres premisas que podríamos encontrar relación con la situación del COVID-19 y el estudio realizado. En primer lugar, el comportamiento es causado por estímulos externos o internos. En este caso el COVID-19 ha sido el estímulo externo e interno que ha generado sentimientos de solidaridad por parte de la población española, ya fuera por haber tenido seres queridos cercanos afectados como por convivir con la desgracia.

En segundo lugar, siguiendo las premisas de Chivenato, el comportamiento es motivado si existe una finalidad o un objetivo. En este caso hemos podido identificar el proyecto NTCPAE con una finalidad clara y concisa que ha impulsado un comportamiento determinado en la población participante.

$Y$, en tercer y último lugar, en todo comportamiento existe siempre un impulso, deseo, necesidad o tendencia, que sirven para designar los motivos de la conducta. En este sentido hemos identificado motivaciones intrínsecas, como la satisfacción personal, y extrínsecas, como influir positivamente en la vida de otros, generar un impacto positivo en su salud mental o romper con la soledad percibida ante una situación de institucionalización.

Como limitaciones del estudio cabe señalar que el cuestionario diseñado ha sido de elaboración propia. En la literatura no hemos encontrado ningún cuestionario validado sobre motivación y solidaridad. A su vez, no hemos encontrado ningún estudio científico que abordara el fenómeno analizado, por lo que ha sido difícil la comparación con otros trabajos. Ante este vacío, consideramos interesante que se profundizara en elaborar herramientas de medición sobre actitudes motivacionales hacia la solidaridad. En contra, consideramos que nos encontramos ante un trabajo inédito, ya que se visibiliza un proyecto solidario, altruista y generado bajo la motivación de la acción comunitaria que podría tener continuidad en futuros proyectos en diferentes contextos sociosanitarios.

\section{Agradecimientos}

Como autora principal de este artículo, en primer lugar, me gustaría agradecer a todo el equipo de "No te conozco pero aquí estoy". Especialmente a Marta Dalmases, 
Paula Martínez, Clara Hurtado e Isabel Kofoed por ayudar con la organización de los datos, el desarrollo y difusión del cuestionario. Agradecer al Doctor MirónGonzález por apoyarme en el desarrollo de este artículo y su implicación en compartir este proyecto que tanto nos ha llenado de ilusión. Muchas gracias.

\section{Referencias bibliográficas}

Antena 3 Noticias. (2020). Mensajes de apoyo de voluntarios a enfermos de coronavirus ingresados: "No te conozco, pero puedo ayudarte». Recuperado de https://bit.ly/3gynhFQ

Arora, G., Kroumpouzos, G., Kassir, M., Jafferany, M., Lotti, T., Sadoughifar, R., Goldust, M. (2020). Solidarity and transparency against the COVID-19 pandemic. Dermatologic Therapy, dth13359. https://doi.org/10.1111/dth.13359

Bauman, Z. (2005). Amor líquido: Acerca de la fragilidad de los vínculos humanos. México, D.F.: Fondo de Cultura Económica.

Bauman, Z. (2019). Modernidad líquida. Buenos Aires, Argentina: Fondo de Cultura Económica.

Castillo del, A. (2020, mayo 1). Cartas anónimas para los enfermos cántabros: "No te conozco, pero estas palabras son para ti». El Diario Montañés. Recuperado de: https://bit.ly/36ETkzn

Chiavenato, I. (2007). Administración de recursos humanos: El capital humano de las organizaciones. Mexico: Mc Graw Hill.

Feito, L. (2007). Vulnerabilidad. Anales del Sistema Sanitario de Navarra, 30, 07-22.

Figueredo, E. (2020, marzo 26). Cuatro enfermeras del Clínic dan a sus pacientes cartas de desconocidos para consolarlos del aislamiento. La Vanguardia. Recuperado de https://bit.ly/2ZNq4Fe

Giraldo Nayrobis, Y., \& Ruiz-Silva, A. (2015). La comprensión de la solidaridad. Análisis de estudios empíricos. Revista latinoamericana de Ciencias Sociales, Niñez y Juventud, 13(2), 609-625. https://doi.org/10.11600/1692715x.1324092614

González Álvarez, M. I. (2010). Comunicación para la solidaridad: Las ONG y el papel de la comunicación social y periodística en la sensibilización de la opinión pública y el desarrollo (Universidad Complutense de Madrid). Recuperado de https://eprints.ucm.es/11633/1/T32381.pdf

Instituto de Salud Carlos III. (2020). Informe sobre la situación de COVID-19 en España (N.o 27). Recuperado de https://bit.ly/3gz9gYn

Martín, M. (2020, marzo 26). Una cadena de favores infinita para golpear al coronavirus: "No te conozco, pero estamos juntos en esto". El País. Recuperado de https://bit.ly/3dki1Uo

Médicos del Mundo. (2012, Mayo 17). Campaña Somos: 25 ONG se unen para agradecer el apoyo de la ciudadanía española. Recuperado de https://bit.ly/2AmJZ2R

Mengin, A., Allé, M. C., Rolling, J., Ligier, F., Schroder, C., Lalanne, L.,Giersch, A. (2020). Conséquences psychopathologiques du confinement. L'Encéphale, S0013700620300750. https://doi.org/10.1016/j. encep.2020.04.007

Ministerio de Defensa. (2018). Panorama de tendencias geopolíticas. Horizonte 2040. Recuperado de Ministerio de Defensa website: https://bit.ly/2ZRyU4H

Ministerio de Sanidad, Política Social e Igualdad. (2011). Diagnóstico de la situación del voluntariado de acción social en España (p. 306). Ciempozuelos (Madrid): Ministerio de Sanidad, Política Social e lgualdad.

Pang, T. (2016). Is the global health community prepared for future pandemics? A need for solidarity, resources and strong governance. EMBO Molecular Medicine, 8(6), 587-588. https://doi.org/10.15252/ emmm.201606337

Plataforma del Voluntariado de España. (2019). La acción voluntaria en 2018 (p. 55). Madrid: Ministerio de Sanidad, Servicios Sociales e Igualdad.

Poulet, M. (2020, abril 24). L'initiative espagnole qui veut redonner espoir aux malades du Covid-19 isolés. France 24. Recuperado de https://bit.ly/2MblSqM

Qian, X., Ren, R., Wang, Y., Guo, Y., Fang, J., Wu, Z.-D., Han, T.-R. (2020). Fighting against the common enemy of COVID-19: A practice of building a community with a shared future for mankind. Infectious Diseases of Poverty, 9(1), 34. https:// doi.org/10.1186/s40249-020-00650-1

Requeijo-Rey, P., Losada-Diaz, J. C., \& García-Jiménez, L. (2015). Directrices europeas para la gestión de crisis sanitarias: Estado de la cuestión. Revista Española de Comunicación en Salud, 6(2), 115-126.

Taylor, S. J., \& Bogdan, R. (1996). Introducción a los métodos cualitativos de investigación: La búsqueda de significados. Barcelona, España: Paidós. 